\title{
LAS ESTRATEGIAS DE LA BENEVOLENCIA (Sobre las relaciones entre el paternalismo y la bioética)
}

\author{
Macario Alemany \\ Universidad de Alicante
}

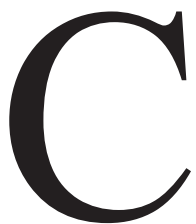

on frecuencia se ha sostenido que el paternalismo es el problema ético fundamental de la práctica médica ${ }^{1}$, aunque esta afirmación puede entenderse de dos maneras bien diferentes. Según una primera interpretación, el paternalismo médico sería el modelo de relación médico-paciente de la medicina tradicional, descrito por Hipócrates y ejercido hasta nuestros días². Un "modelo" que es problemático dadas ciertas asunciones actuales sobre el valor de la autonomía individual y que debe ser sustituido, se propone, por el modelo del consentimiento informado. Mientras que en la concepción tradicional paternalista el punto de partida de las argumentaciones justificantes son la naturaleza, obligaciones y prerrogativas de la profesión médica, en la concepción actual el prius son los derechos del paciente y el derecho del médico pasa a ser un posterius ${ }^{3}$.

Según la segunda interpretación, el paternalismo médico no es únicamente el modelo tradicional de la relación médico-paciente, sino que es una categoría conceptual necesaria para delimitar el problema moral específico de la medicina, cuya solución sería, precisamente, la determinación de las condiciones de justificación de la intervención paternalista. A diferencia de

${ }^{1}$ Véase: por ejemplo, Culver, Charles M. y Bernard Gert: Philosophy in Medicine (Conceptual and Ethical Issues in Medicine and Psychiatry), ed. Oxford University Press, N.Y., 1982, p. 126.

${ }^{2}$ Un muy buen resumen sobre este modelo tradicional puede leerse en la siguiente obra: Gracia, Diego: "Historia de la ética médica", en Vilardell, Francisco: Ética y medicina, Ed. Espasa, Madrid, 1988, pp. 27-65.

${ }^{3}$ Esta crítica del paternalismo médico es análoga a la que John Locke hiciera de las relaciones paterno-filiales: anteponer el derecho del hijo al poder del padre. Bobbio resume esta crítica diciendo que en la obra de Locke el derecho del padre pasa de ser un prius a ser un posterius. Véase: Locke, John: Segundo ensayo sobre el gobierno civil, Ed. Austral, Madrid, 1997, p. 243 y ss.; y, Bobbio, Norberto: Locke e il diritto naturale, ed. G. Giappichelli, Torino, 1963 , p. 241 y ss. 
la perspectiva anterior, en ésta se reconoce que el paternalismo médico no puede ser totalmente superado.

En este artículo, partiré de la segunda interpretación, que implica que el paternalismo en algunos casos está justificado y en otros no. Trataré de poner en relación la categoría del paternalismo con un conjunto de problemas que se suelen tratar bajo la rúbrica de "bioética", al objeto de proporcionar una taxonomía básica de tipos de problemas bioéticos en los que se plantea la cuestión de la justificación de las intervenciones paternalistas. Para ello presentaré, en primer lugar, una caracterización sucinta de lo que cabe entender por "paternalismo" y haré unas breves consideraciones sobre su plausibilidad moral; en segundo lugar, me referiré al contenido de la bioética y a algunas importantes distinciones que, a mi juicio, tiene sentido hacer dentro de la misma y, en tercer lugar, pondré en relación estas distinciones con el paternalismo.

\section{Paternalismo}

El concepto de paternalismo ha sido objeto de una larga y compleja discusión que comienza en el principio de los años setenta y se prolonga durante dos décadas sin arrojar resultados concluyentes ${ }^{4}$. Todas las propuestas conceptuales en torno al paternalismo tienen como mínimo común denominador la que podríamos denominar "analogía paternalista", es decir, una semejanza de relaciones en la que el foro de la analogía lo constituye la bien conocida relación paterno-filial y el tema lo constituye la relación soberano-súbdito, patrón-obrero, médico-paciente, etc. Sin embargo, hay

\footnotetext{
${ }^{4}$ Véase: Dworkin, Gerald: "Paternalism", en Wasserstrom, R. A. (ed.): Morality and the Law, Ed. Wadsworth Publishing Co., Belmont, 1971, pp. 107-126. Éste es, sin duda, el artículo más influyente en la reciente discusión sobre el paternalismo. Existen las siguientes reediciones del mismo: en The Monist 56, nº 1 (1972), pp. 64-84; en Sartorius, Rolf: Paternalism, Ed. University of Minnesota Press, Minneapolis, 1987, pp. 19-34; y en FeinBerg, Joel y Jules ColeMAN: Philosophy of Law, Ed. Wadsworth, Belmont, 2000, pp. 271-280. Existe una traducción del mismo al castellano realizada por Jorge M. Seña e incluida en la colección de ensayos: Derecho y Moral. Ensayos analíticos, editada por Jerónimo Betegón y Juan Ramón de Páramo, publicada en 1990. Véase igualmente: FeInBerg, Joel: The Moral Limits of the Criminal Law. Harm to Self, Ed. Oxford University Press, N.Y. 1986; y FeinBerg, Joel: "Legal Paternalism", en Canadian Journal of Philosophy 1, $\mathrm{n}^{\circ} 1$ (1971), pp. 106-24 (reeditado en Sartorius, Rolf: Paternalism, cit., pp. 3-18); RAwls, John: Teoría de la Justicia, Ed. Fondo de Cultura Económica, México, 1997, pp. 230 y ss.; Gert, Bernard y Charles M. Culver: "Paternalistic Behavior", Philosophy \& Public Affairs 6 (1976), vol. 1, pp. 45-57; Gert, Bernard y Charles M. Culver: "The Justificaction of Paternalism", Ethics 89 (1979), pp. 199-210; Culver, Charles M. y Bernard Gert: Philosophy in Medicine cit., y Gert, Bernard, Charles M. Culver y K. Danner Clouser: Bioethics. A Return to Fundamentals, Ed. Oxford University Press, N.Y., 1997. En lengua castellana, merecen destacarse especialmente las siguientes obras: Garzón Valdés, Ernesto: “¿Es éticamente justificable el paternalismo jurídico?”, en Doxa 5 (1988), pp. 155-173; y Nino, C.S.: Ética y derechos humanos, Ed. Ariel, Barcelona, 1989, pp. 413 y ss.
} 
notables discrepancias sobre cómo construir la analogía, qué definición es la más apropiada y, en particular, sobre qué relevancia darle al carácter peyorativo que en el lenguaje ordinario tiene el término "paternalismo".

No entraré a explicar las diferentes propuestas que se han hecho de definición de "paternalismo", puesto que ello ocuparía demasiado espacio para los objetivos de este artículo. Me limitaré, por tanto, a exponer sucintamente algunas ideas sobre el concepto de paternalismo (al menos, sobre un concepto central de paternalismo).

A mi juicio, aclarar el concepto de paternalismo equivale a completar y aclarar el significado de la siguiente expresión:

E1: El agente A ejerce paternalismo sobre el sujeto B

Este enunciado presupone que el paternalismo consiste básicamente en una relación entre dos sujetos. Se trataría de una relación caracterizada por los siguientes elementos:

a) Los sujetos de la relación paternalista (A y B)

b) El modo de relación paternalista

c) La finalidad paternalista

Empecemos por este último elemento. Lo que caracteriza al genuino paternalismo es su finalidad benevolente. Éste sería, puede decirse, el elemento positivo de la acción paternalista. Si el agente A no trata honestamente de beneficiar a B, no estaríamos ante un caso de paternalismo, sino, en algunas ocasiones, ante un simulado paternalismo. Un ejemplo muy significativo de este falso paternalismo sería el de la explotación por parte de Leopoldo II del denominado Congo belga ${ }^{5}$. Muchas de las críticas a la conducta paternalista parten de supuestos de falso paternalismo. Pero, obviamente, el paternalismo que puede plantear una genuina duda sobre su justificación es aquel que realmente se orienta a beneficiar al sujeto B.

Por tanto, una parte del significado de E1 se expresaría por medio del siguiente enunciado:

E2: El agente paternalista A trata de beneficiar al sujeto B

"Beneficiar" es, a su vez, una palabra ambigua y que remite a conceptos con una notable zona de penumbra. Por lo que se refiere al paternalismo, habría que hacer dos distinciones fundamentales. La primera de ellas sería la distinción entre beneficios físicos, psíquicos o económicos, por un lado, $\mathrm{y}$ beneficios morales, por otro. Siguiendo a Hart, creo que el paternalismo se caracteriza por perseguir el primer tipo de beneficios y que la persecución de beneficios morales caería bajo la categoría de lo que suele denominarse

\footnotetext{
${ }^{5}$ Véase: Hochschild, Adam: El fantasma del rey Leopoldo (Codicia, terror y heroísmo en el África colonial), Ed. Península, Barcelona, 2002.
} 
"perfeccionismo". En segundo lugar, dentro del concepto de beneficiar podría distinguirse entre "beneficiar en sentido estricto" y "evitar daños". Esta distinción no es conceptualmente fácil y no entraré ahora a discutir sus pormenores. Por "beneficiar en sentido estricto" entiendo promover los intereses de un individuo más allá de un nivel básico de satisfacción de los mismos y por "evitar daños" entiendo evitar que los intereses del individuo caigan por debajo de ese nivel o, cuando ya se hallan por debajo, llevarlos hasta, o por encima, del mismo. Curar a una persona enferma sería un claro ejemplo de "evitar daños" y también prevenir la enfermedad, mientras que un "tratamiento" de belleza sería un ejemplo de "beneficiar en sentido estricto". Algunos autores han sostenido que cuando se trata de imponer beneficios psíquicos, físicos o económicos ya no se trata de paternalismo sino de una figura afín 7 , mientras que otros han señalado que se trataría de un tipo de paternalismo, extremo o radical $^{8}$. No obstante, podría decirse que, en el uso más extendido de "paternalismo", el enunciado E2 equivaldría al siguiente:

E3: El agente A trata de evitar daños físicos, psíquicos o económicos al sujeto B

Si el paternalismo pretende evitar daños, la pregunta que surge es por qué se entiende generalmente que hay un problema de justificación en la acción paternalista. La razón de ello tiene que ver con el segundo elemento de la relación paternalista: el modo. Del paternalismo se ha dicho que consiste en imponer coactivamente un comportamiento, interferir con la libertad, engañar, violar una norma moral con respecto al sujeto B, etc. Dar con el modo propio de la acción paternalista, que lo distinguiría del resto de acciones benevolentes o altruistas, es el núcleo de la discusión sobre el paternalismo. A mi juicio, convendría distinguir aquí entre el modo y el medio de la acción paternalista ${ }^{9}$. Es imposible señalar en abstracto qué medios son idóneos para ejercer paternalismo; ello depende de las circunstancias concretas del caso. Sin embargo, sí que habría un modo propio de la acción paternalista que consistiría en oponerse a las preferencias del sujeto B en el momento de la intervención paternalista. Por ello se suele decir que el paternalismo

${ }^{6}$ Véase: Hart, H.L.A.: Law, Liberty and Morality, Ed. Stanford University Press, 1963, p. 31. Seguramente, Hart fue el primero en introducir el término "paternalismo"en la discusión académica sobre los límites morales del Derecho penal (al objeto de distinguirlo del "perfeccionismo"). Hart considera que el paternalismo consiste en "proteger a los individuos de sí mismos".

${ }^{7}$ Véase: GARZÓN VALDÉs, Ernesto: “ES éticamente justificable el paternalismo jurídico?”, en Doxa, 5 (1988), p. 157.

${ }^{8}$ Véase: FeinBerg, Joel: Harmless Wrong-Doing, Ed. Oxford University Press, N.Y., 1990, p. 312.

${ }^{9}$ Debo agradecer a Daniel González Lagier que me llamara la atención sobre la oportunidad de hacer esta distinción. 
trata de proteger a los individuos de sí mismos, de imponer prudencia, etc. Si un paciente, por ejemplo, prefiere no continuar con un tratamiento que es necesario para la curación, sería paternalista conseguir, por cualquier medio (engañando, imponiéndolo por la fuerza, manipulándolo, etc.), que continúe con el mismo. Podemos, por tanto, completar nuestro primer enunciado E1 diciendo que éste sería equivalente al siguiente:

E4: El agente A trata de evitar daños físicos, psíquicos o económicos al sujeto $B$, contra las preferencias de $B$ en el momento de la intervención paternalista

Por último, tenemos los sujetos de la relación. A partir de este elemento, podríamos diferenciar algunos tipos principales de paternalismo: 1) paternalismo en la empresa ${ }^{10}$, 2) paternalismo estatal, 3) paternalismo entre Estados $^{11}$ y 4) paternalismo médico. A cada uno de los anteriores tipos le correspondería la siguiente expresión equivalente: 1) "el patrón ejerce paternalismo sobre el empleado", 2) "el Estado ejerce paternalismo sobre los ciudadanos", 3) "el Estado $A$ ejerce paternalismo sobre el Estado B", y 4)

${ }^{10}$ El término "paternalismo" (cuyos primeros usos constatados se remontan al último tercio del siglo XIX, siendo originario de la lengua inglesa) se usó en lengua castellana a principios de siglo casi exclusivamente para referirse a este tipo de paternalismo. Es de destacar que, por ejemplo, en la Enciclopedia Universal Ilustrada de Espasa, publicada por primera vez en 1920, la única acepción que se incluye de "paternalismo" es, precisamente, la siguiente: "Régimen de relaciones entre el patrono y el obrero que, asimilándolas a las familiares, atribuye al patrono una especie de protección tutelar sobre sus obreros". Véase: Voz "Paternalismo" en Enciclopedia Universal Ilustrada, ed. Espasa-Calpe, Madrid, 1966, t. 42. Curiosamente, por los mismos años en que en la filosofía moral y jurídica se "pone de moda" hablar del paternalismo (a principios de la década de los setenta), en la historiografía social irrumpe con fuerza esta categoría para analizar no sólo el denominado "paternalismo industrial", sino también las relaciones socio-económicas de la historia anteriores al capitalismo. Los ejemplos históricos de paternalismo industrial más importantes son las llamadas ciudades-fábrica. Paradigmáticos son los casos de Pullman City en los Estados Unidos, la cité Mulhouse o Le Creusot en Francia, Le Grand Hornu en Bélgica, etc. En España cabe destacar el paternalismo practicado en las explotaciones mineras de Río Tinto en Huelva y en las empresas mineras asturianas como Hullera Española en Bustiello, Solvay en Lieres o Ensidesa en Avilés. Los patronos paternalistas iban mucho más allá del pago del salario y se comprometían a proveer a los obreros de economatos, viviendas, cajas de previsión, educación, servicios religiosos, pero siempre supeditado a la continuidad en la empresa y al buen rendimiento en el puesto de trabajo. Se discute si se trataba realmente de paternalismo o simplemente de una manera de disciplinar a la mano de obra (sacar al obrero de la taberna era una obsesión de la época) y asegurar su reproducción. A mi juicio, sin embargo, en círculos de lo que podríamos llamar catolicismo social se predicaba y practicaba un paternalismo bastante sincero (no del todo porque con la filantropía se mezclaba con el miedo al movimiento obrero). Sobre el paternalismo industrial en España, véase: SiERRA Álvarez, José, El obrero soñado. Ensayo sobre el paternalismo industrial (Asturias, 18601917), Ed. Siglo XXI, Madrid, 1990; Babiano Mora, J.: Paternalismo industrial y disciplina fabril en España, 1938-1958, Ed. CES, Madrid, 1998.

${ }^{11}$ Véase: GARZón VALDÉs, Ernesto: “Intervencionismo y paternalismo”, en GARZón VALDÉs, Ernesto: Derecho, ética y política, Ed. CEC, Madrid, 1993, pp.379-399. 
"el médico ejerce paternalismo sobre el paciente y/o sus allegados". Aquí nos interesa en particular este último tipo de paternalismo, aunque también, como veremos, es relevante para la bioética el paternalismo estatal. Vale la pena mencionar que entre estas categorías de paternalismo se dan diferencias en cuanto a los medios de que preferentemente se vale la intervención y las finalidades específicas. Al paternalismo estatal, por ejemplo, se le suele adscribir como medio preferente la coacción y como finalidad la más genérica del bienestar (de ahí la calificación de paternalista que se hace del Estado del Bienestar), mientras que al paternalismo médico se le suele adscribir como medio preferente el engaño o la ocultación de información (lo que explica que en este ámbito se denomine al consentimiento válido "consentimiento informado") y como finalidad específica la salud del sujeto B (bienestar físico o psíquico).

A todas estas categorías de paternalismo se le superpone la de paternalismo jurídico, que se identifica con frecuencia con la de paternalismo estatal. A mi juicio, sin embargo, no todo paternalismo estatal es paternalismo jurídico y tampoco creo que todo paternalismo jurídico sea paternalismo estatal. Sin entrar ahora en este problema, aquí tan sólo quisiera apuntar la tesis de que no se puede presuponer sin más que el paternalismo médico no es paternalismo jurídico (puesto que el médico puede actuar de acuerdo con un permiso o autorización del Estado) y que, en todo caso, plantea un problema de relevancia jurídica (con frecuencia formulado como el problema de "cuáles son los derechos de los pacientes").

El anterior análisis tiene algunos presupuestos que conviene hacer explícitos. En primer lugar, el agente A debe creer que: 1) Él sabe mejor que B lo que le conviene a B, y 2) que B es, no obstante, capaz de formar una preferencia contraria a la acción paternalista. En segundo lugar, B debe creer que él sabe mejor que A lo que le conviene a sí mismo (que él es el mejor juez de sus propios intereses).

De acuerdo con esta aproximación conceptual, se podrían proponer los siguientes ejemplos de paternalismo: 1) administrar un placebo a un paciente para reducir su ansiedad; 2) inmovilizar a un paciente contra su voluntad por medio de ataduras o drogas, para evitar que se autolesione; 3) hacer una transfusión de sangre al paciente contra su voluntad, necesaria para salvar su vida; 4) no informar al paciente de la gravedad de su diagnóstico, a pesar de que éste demanda información completa, para evitar una depresión suicida; 5) no dispensar medicamentos peligrosos sin receta médica; etcétera.

Las dificultades teóricas en torno al concepto de paternalismo guardan a mi juicio una estrecha conexión con las dificultades teóricas en torno al concepto de poder. Hasta el punto de que podría afirmarse que la relación paternalista es siempre una relación de poder y que la acción paternalista 
es siempre un acto de poder. Algunos autores han señalado que se ejerce poder sobre una persona siempre que se consigue que esa persona haga lo que de otra manera no habría hecho. La cuestión que me parece crucial para la discusión del paternalismo es si es posible que el agente A no sólo logre imponer su voluntad sobre B (lo cual muestra que tiene poder sobre B), sino que también logre beneficiarlo de esta manera. Algunos sostendrán que esto no es posible, porque el interés de B en su autonomía siempre pesará más que cualquier otro interés que $\mathrm{B}$ tenga y que es promovido o protegido por la intervención paternalista. Sin embargo, a mi juicio, esto último no es cierto y, por tanto, son posibles ejercicios paternalistas del poder que sean realmente beneficiosos (que promuevan los intereses de B considerados en su conjunto) para los individuos tratados paternalistamente ${ }^{12}$.

Pues bien, uno de los ámbitos donde creo que hay más ocasiones para ejercer un honesto y exitoso paternalismo es en el de la bioética. La asimetría del conocimiento entre los médicos y sus pacientes, las circunstancias que suelen acompañar a la enfermedad y el compromiso sincero de los profesionales de la salud con el bienestar de los pacientes hacen que se generen situaciones en las que es plausible el paternalismo. Ello no implica negar que el paternalismo sea problemático desde el punto de vista moral, puesto que no sólo valoramos el bienestar sino también, y de una manera muy especial, la autonomía individual. Hay que juzgar a la luz de las circunstancias de los casos, partiendo del reconocimiento de estos valores últimos (bienestar y autonomía), qué intervenciones paternalistas están justificadas y cuáles no. Más que decir a los médicos que nunca han de ser paternalistas, habría que decirles, a mi juicio, que nunca han de serlo si no están en condiciones de justificar su acción, es decir, de dar razones que apoyen la decisión tomada ${ }^{13}$.

${ }^{12}$ Sobre la racionalidad de preferir menos libertad (u oportunidades) a más libertad (u oportunidades) para proteger otros bienes, véase: HART, H. L. A.: "Rawls on Liberty and Its Priority”, en Daniels, Norman (ed.): Reading Rawls, Ed. Basic Books, Nueva York, 1975, pp. 249-252; Elster, Jon: Ulises y las Sirenas (Estudios sobre racionalidad e irracionalidad), Ed. FCE, México, 1997, pp. 66-188; y ElsTER, Jon: Ulises desatado (Estudios sobre racionalidad, precompromiso y restricciones), Ed. Gedisa, Barcelona, 2002.

${ }^{13}$ Bernard Gert ha señalado que la justificación del paternalismo (y, en general, la justificación moral) requiere de una actitud de imparcialidad, de acuerdo con la cual si creemos que nuestra acción concreta está justificada debemos creer, igualmente, que estaría justificado permitir públicamente esa acción. Lo contrario de esta actitud imparcial es, señala Gert, la "arrogancia" de creer que la propia acción está justificada, pero no se puede defender públicamente que cualquiera actuaría justificadamente de la misma manera en las mismas circunstancias. Esta actitud "arrogante" es frecuente en las situaciones en las que se suelen dar comportamientos paternalistas: por ejemplo, es frecuente que médicos que practican la eutanasia (en la "privacidad" de su unidad) y que creen que hacen lo correcto, sin embargo, consideren inaceptable 
Por ello me parece importante indagar contextos de plausible paternalismo y, en este artículo, distinguir diferentes situaciones para el paternalismo que son tratadas conjuntamente bajo la rúbrica de la bioética.

\section{Bioética}

El término "bioética" comenzó a usarse en los años setenta y con él se suele hacer referencia a los desafíos éticos planteados por el desarrollo de las ciencias biológicas y médicas ${ }^{14}$. No es fácil determinar los contornos del objeto de estudio de esta disciplina. El prestigioso instituto The Hastings Center, dedicado a la investigación en bioética, señala que su objetivo es "explorar las cuestiones fundamentales y emergentes en la asistencia sanitaria (health care), biotecnología y medio ambiente"15. Los proyectos de investigación que se desarrollan actualmente en el instituto se agrupan bajo cinco categorías principales: 1) genética y biotecnología, 2) asistencia sanitaria y política sanitaria, 3) ética, ciencia y medio ambiente, 4) internacional (donde se investigan temas como el VIH/SIDA en África oriental o la medicina y el mercado), y 5) ética e investigación científica ${ }^{16}$.

Si el objeto de estudio de la bioética es, como vemos, muy amplio y de fronteras imprecisas, la naturaleza misma de la disciplina también parece difícil de delimitar. Desde sus orígenes y a lo largo de los años setenta, se enfrentaron filósofos y médicos en dos interpretaciones opuestas de la bioética: para los filósofos, esta disciplina era una aplicación más de la ética, que requería de los médicos o investigadores tan sólo la aportación de los datos; para los médicos e investigadores, sin embargo, la bioética era un conocimiento esencialmente médico y científico, donde eran los filósofos los que podían ser llamados a aportar materiales ${ }^{17}$. Ambas posturas extremas fueron pronto superadas y puede decirse que encontraron su síntesis en una concepción procedimental o metodológica de la bioética, según la cual la misión principal de ésta es dotar a los médicos e investigadores de un método apto para obtener soluciones a los problemas éticos de su práctica profesional (generalmente, por medio de la elaboración de protocolos de actuación) ${ }^{18}$.

que públicamente se permita. Véase: Gert, Bernard, Charles M. Culver y K. Danner Clouser: Bioethics. A Return to Fundamentals, cit, p. 43.

${ }^{14}$ Gracia, Diego: Procedimientos de decisión en ética clínica, Ed. Eudema, Madrid, 1991, p. 25.

${ }^{15}$ Véase la página web principal del instituto en http://www.thehastingscenter.org/

${ }^{16}$ Ibídem. Una visión general de los problemas abordados por la bioética se puede obtener consultando el Hastings Center Report, publicado desde 1971 o la Bibliography of Bioethics, editada por L. Walters y T. J. Tamar anualmente desde 1975.

${ }^{17}$ Gracia, Diego: Procedimientos de decisión en ética clínica, cit., p. 25.

18 Aquí "procedimental" no se debe entender en el sentido de "justicia procesal". Los procedimientos de decisión en bioética están diseñados para dar con la solución correcta a un 
Esta dimensión metodológica de la bioética es sobresaliente en el enfoque de los principios, que hoy podríamos considerar la teoría bioética más importante, desarrollado en su versión estándar por Tom L. Beauchamp y James F. Childress ${ }^{19}$ y también en otras concepciones de la bioética: por ejemplo, el casuismo defendido por Jonsen y Toulmin ${ }^{20}$. En definitiva, como ha destacado Manuel Atienza, la bioética podría entenderse como una parte de la teoría de la argumentación ${ }^{21}$.

\section{Algunas distinciones importantes}

Al objeto de aclarar las relaciones de la bioética con el paternalismo, es útil distinguir, dentro del amplio conjunto de problemas que se tratan bajo la rúbrica de "bioética", entre los siguientes tipos de acciones o intervenciones:

a) Acciones en el contexto de la investigación, en particular la experimentación con seres humanos, y en el contexto de la práctica médica;

b) Acciones orientadas a evitar enfermedades y las orientadas a producir beneficios positivos;

c) Acciones preventivas y curativas;

d) Intervención genética y otras intervenciones médicas.

\subsection{Investigación y práctica}

La distinción entre investigación y práctica suele verse reflejada en las definiciones mismas de "bioética", que se presenta como compuesta inicialmente por estos dos ámbitos de problemas éticos. En opinión de los redactores del influyente Informe Belmont, orientado a establecer los principios éticos que deben guiar la experimentación con seres humanos, la distinción entre investigación y práctica es "borrosa" por dos razones: primera, porque en ocasiones investigación y práctica se dan simultáneamente (por ejemplo, cuando la investigación está orientada a evaluar una terapia) y, segunda, porque a terapias que son desviaciones de la práctica comúnmente aceptada se les suele denominar "experimentales", constatándose, además, incertidum-

caso, pero no definen la "solución correcta" como "aquélla que es el resultado de seguir los pasos del procedimiento".

${ }^{19}$ Véase: Beauchamp, Tom L. y James F. Childress: Principios de Ética Biomédica, Ed. Masson, Barcelona, 2002.

${ }^{20}$ Véase: Jonsen, Albert R. y Stephen Toulmin: The Abuse of Casuistry: A History of Moral Reasoning, Ed. University of California Press, Berkeley, 1988. Véase, igualmente: Toulmin, Stephen: "De qué manera la medicina le salvó la vida a la ética", en Análisis filosófico, vol. XVII, 2 (1997), pp. 119-135.

${ }^{21}$ Véase: AtıEnZa, Manuel: "Juridificar la bioética", en VÁzQUEZ, Rodolfo (comp.): Bioética y Derecho (Fundamentos y problemas actuales), Ed. Itam-FCE, México. 
bre sobre la distinción entre "experimental" e "investigación”. Sobre cómo trazar la distinción entre investigación y práctica dicen lo siguiente:

"En la mayoría de casos, el término "práctica" se refiere a intervenciones cuyo fin es acrecentar el bienestar de un paciente individual o de un cliente, y hay motivos razonables para esperar el éxito. El fin de la práctica médica es ofrecer un diagnóstico, un tratamiento preventivo o una terapia a individuos concretos. Como contraste, el término "investigación" denota una actividad destinada a comprobar una hipótesis, que permita sacar conclusiones y, en consecuencia, contribuya a obtener un conocimiento generalizable (expresado, por ejemplo, en teorías, principios, y enunciados de relaciones). La investigación se describe generalmente en un protocolo formal que presenta un objetivo y un conjunto de procedimientos diseñados para alcanzar este objetivo"22.

Este párrafo muestra que el interés de la comisión no era sólo distinguir entre investigación y práctica (lo cual es relativamente fácil pues, como señalan, el fin de ambas actividades es diferente), sino sobre todo aclarar por qué es moralmente importante distinguirlas. De ahí que se insista en dos aspectos de la práctica: a) que beneficia generalmente al individuo que se le aplica, y b) que suele haber motivos razonables para esperar el éxito del tratamiento; características de las que carecería, a su juicio, la investigación y, en particular, la investigación que se hace por medio de la experimentación con seres humanos. No obstante, reconocen que la práctica no siempre beneficia al sujeto que es intervenido, puesto que, en ocasiones, beneficia sólo a otro (por ejemplo, el donante en una transfusión de sangre) o beneficia al sujeto intervenido y a otros (por ejemplo, una vacuna que evita contagiar y ser contagiado). En cuanto a las prácticas que son muy innovadoras, por lo que se pueden denominar "experimentales", el comité advierte que no son "investigación", puesto que les faltaría el aspecto formal propio de la misma. Aunque el comité no lo diga expresamente, lo relevante desde el punto de vista moral de estas prácticas "experimentales" es que se apartarían de la práctica estándar en el grado de confianza en obtener el éxito.

\subsection{Tratamiento y mejora}

Dentro del objetivo genérico de promover el bienestar físico y psíquico, se le suele otorgar una gran relevancia moral a la distinción entre el fin del tratamiento de la enfermedad (intervenciones negativas) y el de mejorar la salud (intervenciones positivas). Esta distinción es utilizada fundamentalmente por los seguros médicos, tanto públicos como privados, para diferenciar las prestaciones que deben ser cubiertas de aquéllas que deben ser

${ }^{22}$ National Comisión for the Protection of Human Subjects of Biomedical and Behavioral Research: Informe Belmont (30 de septiembre de 1978), ed. Observatori de Bioètica i Dret, http://www.ub.es/fildt/bioetica.htm. 
costeadas por el propio asegurado. Además, en ocasiones, sirve igualmente para delimitar lo que es permisible hacer a los individuos ${ }^{23}$.

Estos dos tipos de intervenciones, negativas y positivas, se corresponden con la distinción conceptual entre "evitar daños" y beneficiar". Antes hemos señalado que puede decirse que si bien "evitar daños" sería una forma de beneficiar en sentido genérico, cuando ambos términos se contraponen se ha de entender por "beneficiar" promover los intereses de un individuo más allá de un nivel básico de satisfacción de los mismos y por "evitar daños" impedir que estos intereses caigan por debajo de este nivel básico o llevarlos de vuelta al mismo. El problema es, por supuesto, cómo determinar el nivel básico de satisfacción. En el caso que nos ocupa, la bioética, la noción de enfermedad (en un sentido amplio, que incluiría la discapacidad, lesiones, heridas, etc.) puede entenderse precisamente como un instrumento para determinar este nivel. Ahora bien, la noción de enfermedad no es, en absoluto, fácil.

Es plausible sostener que las enfermedades, en sentido amplio, son un mal y, en más ocasiones de las que se suele pensar, un mal evitable, es decir, males que son consecuencias previsibles de las acciones u omisiones de los seres humanos ${ }^{24}$. En ocasiones, alguna enfermedad puede adquirir el carácter de "mal teológico"25, algo contra lo que el hombre no puede luchar, como las "catástrofes naturales", pero, generalmente, son evitables, tratables o las dos cosas. Algunas enfermedades pueden ser evitadas, además, por el propio sujeto que las padece, lo cual no implica por sí sólo que se deba culpabilizar a estos enfermos o que no se les deba prestar ayuda ${ }^{26}$. Otras, la mayoría, pueden ser evitadas o curadas con políticas públicas que pongan al alcance de los afectados los medios preventivos disponibles o que proporcionen los tratamientos también disponibles o, incluso, que fomenten la investigación para las enfermedades todavía sin tratamiento. La propagación del SIDA en

${ }^{23}$ Sobre la relevancia moral de esta distinción puede encontrarse un buen análisis en: Allen Buchanan, Dan W. Brock, Norman Daniels y Daniel Wikler: Genética y Justicia, Ed.Cambridge University Press, Madrid, 2002, cap. 4, pp. 97 y ss.

${ }^{24}$ Se trata de lo que Joel Feinberg denomina "males legislativos" y que contrapone a los "males teológicos". Véase: FeInBerg, Joel: Harmless Wrong-doing, cit., p. 18.

${ }^{25}$ Utilizo la expresión de Joel Feinberg. Véase: FeinBerg, Joel: Harmless Wrong-doing, cit., p. 18.

${ }^{26}$ Para Joel Feinberg, no son lo mismo "males" y "daños" (en sentido normativo). Hay males, estados de cosas que son lamentados, que no son daños, el resultado de infligir injustamente un mal, por ejemplo los "daños" (en sentido descriptivo) que son infligidos a un individuo con su consentimiento. Las enfermedades pueden ser tanto males que son daños en sentido normativo (por ejemplo, el daño que inflige un sujeto que contagia de SIDA a otro contra su voluntad) como males que son daños en sentido descriptivo (por ejemplo, el contagio del SIDA por un sujeto que ha mantenido voluntariamente, válidamente, relaciones con un seropositivo). 
el tercer mundo es un buen ejemplo de este tipo de "males evitables" que son las enfermedades: pese a las descripciones que de esta propagación se hacen como de "plaga bíblica", no tiene nada de "teológica", puesto que no se están distribuyendo los medicamentos ya disponibles para tratar la enfermedad.

La ausencia de enfermedades parece constituir uno de esos intereses de los seres humanos (intereses del bienestar, necesidades básicas o bienes primarios) que forman una cadena entre sí que no es más fuerte que el más débil de sus eslabones y de cuya satisfacción depende la satisfacción de cualesquiera otros intereses ${ }^{27}$. Muchos autores, a pesar de sostener una concepción subjetivista de los intereses, se han sentido inclinados a admitir que lo que promueve los intereses de bienestar es bueno para los individuos "en cualquier caso, sean cuales sean sus creencias y deseos" ${ }^{28}$, de donde se desprendería su carácter de intereses objetivos de los seres humanos.

Junto a la idea de que la enfermedad es un mal objetivo, las otras dos connotaciones centrales del concepto son la de "anormalidad" y la de "condición del sujeto" 29 . La enfermedad es siempre una condición del propio sujeto que se aparta de lo que es característico en la especie y que, además, es un mal. Con estos elementos las definiciones de "enfermedad" oscilan desde las que pretenden introducir un concepto científico y sin elementos valorativos hasta las que parten, precisamente, de los elementos evaluativos o normativos. En uno de los extremos de la escala, encontraríamos, por ejemplo, la definición de "enfermedad" como "desviaciones adversas o disfunciones respecto a la organización funcional normal típica de la especie o funcionamiento normal"30. Aquí el elemento esencialmente valorativo, la noción de mal, es sustituido por la idea de "disfunción"; la pretensión es que lo que sea una "disfunción o enfermedad" es determinado en el contexto "relativamente objetivo y no evaluador"31 proporcionado por

\footnotetext{
${ }^{27}$ Véase: Rescher, Nicholas: Welfare: The Social Issue in Philosophical Perspective, Ed. University of Pittsburgh Press, Pittsburgh, 1972, p. 6.

${ }^{28}$ FeinBerg, Joel: Harm to Others, cit., p. 40.

${ }^{29}$ Véase: nota 31.

${ }^{30}$ Se trata del concepto de enfermedad propuesto por Christopher Boorse, desarrollado y ampliado por Norman Daniels, y manejada a lo largo de toda la obra Genética y Justicia (obra por la que se cita). Véase: Allen Buchanan, Dan W. Brock, Norman Daniels y Daniel WikLer: Genética y Justicia, cit., pp. 67, 113-115; Boorse, Christopher: "Health as a Theoretical Concept", en Philosophy of Science, 44 (1977), pp. 542-573, "Wright on Functions", en Philosophical Review, 85 (1976), núm. 1, pp. 70-85, "On the Distinction between Disease and Illness", Philosophy and Public Affairs, 5 (1975), núm. 1, pp. 49-68; DAniels, Norman: Just Health Care, Cambridge University Press, Cambridge, 1985.

${ }^{31}$ Allen Buchanan, Dan W. Brock, Norman Daniels y Daniel Wikler: Genética y Justicia, cit., p. 113.
} 
las ciencias biomédicas y veterinarias. En el otro extremo de la escala, se situarían definiciones que, como la propuesta por Bernard Gert y Charles M. Culver, defienden un concepto de enfermedad fuertemente basado en su caracterización primaria como un "mal". A su juicio, "una persona está enferma si y sólo si está en una condición, que no consiste en sus creencias y deseos racionales, tal que sufre, o se incrementa su riesgo de sufrir, un mal (muerte, dolor, discapacidad, pérdida de libertad u oportunidad o pérdida de placer) en ausencia de una causa distinta que esté produciendo esos efectos (distinct sustaining cause)"32. Para estos autores, el concepto de enfermedad se caracteriza por ser normativo, de validez universal y aplicable tanto a las enfermedades físicas como mentales.

Frente a estas aproximaciones al concepto de enfermedad que podrían denominarse "objetivas", se hallan las que señalan su carácter idiosincrásico, el cual hace que lo que sea enfermedad es relativo a un determinado tiempo y lugar. Con frecuencia se sostiene, además, que esta noción es relativa por ser normativa, aunque, como acabamos de ver, nada impide, desde una concepción objetivista de la ética ${ }^{33}$, proponer un concepto de enfermedad normativo pero de validez universal.

Sobre la relevancia moral de la noción de enfermedad me parece que pueden adoptarse tres posturas principales: En primer lugar, partir de una noción relativista o idiosincrásica de la misma, de la cual no podemos extraer juicios de valor universales sobre los que fundar obligaciones o permisiones morales de validez también universal. En segundo lugar, partir de una concepción científica, objetiva y descriptiva, de la cual no podemos extraer juicio de valor alguno (para lo que sería necesario haber introducido

${ }^{32}$ Culver, Charles M. y Bernard Gert: Philosophy in Medicine (Conceptual and Ethical Issues in Medicine and Psychiatry), ed. Oxford University Press, N.Y., 1982. p. 81. Esta definición es un instrumento de análisis conceptual que requiere de algunos comentarios para ser comprendida. En primer lugar, la idea de "condición" se refiere a un estado del sujeto que ya forma parte de él, cualquiera que haya sido su origen, y que, por tanto, ya no puede ser eliminado simplemente cambiando su entorno físico o social (una persona que sufre dolor porque otra le esta doblando la muñeca no está enferma, puesto que el dolor desaparece cuando se afloja la presión sobre la articulación). En segundo lugar, la cláusula de exclusión, "que no consiste en sus deseos y creencias racionales", descarta como enfermedad, por ejemplo, el dolor experimentado por un hombre a causa de la muerte reciente de su mujer o el incremento de riesgo que supone practicar el alpinismo. El último inciso "en ausencia de una distinta causa eficiente" refuerza la idea de la enfermedad como "condición del sujeto", descartando como enfermedad otras situaciones que causan un mal o el incremento del riesgo de sufrirlo: por ejemplo, conducir un coche por la autopista incrementa el riesgo de sufrir un mal pero no es una enfermedad (el riesgo siempre se incrementa cuando se conduce y se reduce cuando no se conduce).

${ }^{33}$ Aquí por "objetivista" me refiero en sentido amplio a todas las teorías éticas que sostienen la validez universal de los valores morales y no sólo a las que defienden su existencia como entidades independientes. 
previamente en la noción de enfermedad un elemento normativo, dejando de ser el concepto puramente descriptivo). Finalmente, partir de una noción normativa y que pretenda validez universal. Esta última postura es, a mi juicio, la más plausible y es la única que nos permite apoyarnos en la distinción entre tratamiento y mejora para alcanzar conclusiones normativas.

En relación con lo anterior, algunos autores (entre ellos, por ejemplo, Ernesto Garzón ${ }^{34}$ o el ya mencionado Bernard Gert $^{35}$ ) han tratado de fundamentar la existencia de una asimetría epistemológica entre los conceptos de lo malo y lo bueno. En opinión de estos autores, las propiedades moralmente relevantes de lo que, desde diferentes sistemas morales, se ha considerado un mal parecen coincidir, mientras que las propiedades moralmente relevantes de lo que se ha considerado, desde diferentes sistemas morales, un bien divergen notablemente.

\subsection{Prevención y curación}

Generalmente, se considera que en la lucha contra las enfermedades hay dos estrategias básicas: la prevención y la curación o tratamiento. Además, a la prevención se le suele considerar una estrategia más racional, aunque en algunos casos pueda darse que ocurra lo contrario; al menos, se puede afirmar que ceteris paribus es mejor evitar una enfermedad que curarla cuando ya se ha originado ${ }^{36}$. La prevención consiste en evitar que se origine una enfermedad y la curación o tratamiento en hacerla desaparecer o en paliar, en la medida de lo posible, sus efectos perjudiciales. A cada una de estas actuaciones le corresponden una serie de rasgos típicos que son, como veremos, relevantes para el discurso moral.

En primer lugar, mientras que la curación o tratamiento es llevado a cabo directamente por profesionales de la salud ${ }^{37} \mathrm{y}$, en particular, por médicos, los

\footnotetext{
${ }^{34}$ Véase: GARZÓn VALDÉS, Ernesto: “¿Puede la razonabilidad ser un criterio de corrección moral?”, en DOXA, 21(1998), vol. II., pp. 145-166.

${ }^{35}$ Véase: Gert, Bernard: The Moral Rules (A New Rational Foundation for Morality), Ed. Harper \& Row, Nueva York, 1970.

${ }^{36}$ Hay que advertir que en el ámbito de la salud pública, con respecto a enfermedades de escasa incidencia, los costes de la prevención para todos pueden superar con mucho los costes del tratamiento de unos pocos afectados. En relación con un individuo aislado, también en ocasiones la prevención puede presentar más inconvenientes que el tratamiento: por ejemplo, puede ser mejor afrontar el tratamiento de una enfermedad contagiosa que sólo se pasa un vez, sin riesgos considerables, que esforzarse en no contraerla. Por eso conviene mantener la cláusula ceteris paribus al afirmar que es mejor la prevención que el tratamiento.

${ }^{37}$ La curación o el tratamiento también depende de que haya los medios disponibles, lo cual atañe, desde luego, a las autoridades. Aquí tan solo quiero destacar que la relación médico-paciente se constituye típicamente en torno al tratamiento y dentro de la institución hospitalaria.
} 
agentes de la prevención son diversos. La prevención atañe, desde luego, a los médicos, pero también directamente a las autoridades, a los agentes económicos y a los ciudadanos mismos. El lugar por excelencia de la curación es el hospital, mientras que la prevención se lleva a cabo en cualquier lugar: en el espacio público, el lugar de trabajo o el domicilio.

En segundo lugar, en relación con lo anterior, cuando la ausencia de prevención deriva en enfermedad se plantea un problema específico sobre la responsabilidad de la no-prevención (¿quién es responsable?) y de las consecuencias que han de seguirse de la misma. El avance en el conocimiento etiológico ha descubierto que, en los países desarrollados, las enfermedades que producen mayor mortalidad prematura (las enfermedades cardiovasculares y el cáncer) tienen su causa principal en los hábitos de vida no saludables de la población: la vida sedentaria, la mala alimentación o el tabaco son los ejemplos más relevantes de estos malos hábitos ${ }^{38}$. Para algunos, este hecho debe servir para afirmar la responsabilidad de los propios ciudadanos en su enfermedad y de aquí derivan que los demás no deben ser obligados a contribuir a su curación ${ }^{39}$; para otros, si bien es verdad lo primero, de ahí no se sigue lo segundo; otros niegan que sean los ciudadanos los responsables y afirman que la responsabilidad corresponde a, por ejemplo, las industrias tabaqueras, las empresas de comida rápida, la televisión $\mathrm{o}$, incluso, las autoridades.

Finalmente, si en la curación misma las obligaciones y prohibiciones jurídicas no juegan un papel directamente relevante, en la prevención, por el contrario, estas normas jurídicas de mandato pueden constituirse en el instrumento principal: por ejemplo, obligando a los ciudadanos a un comportamiento prudente.

\subsection{Genética y otras intervenciones médicas}

El desarrollo de la investigación genética, cuya manifestación más importante es el Proyecto Genoma Humano, abre el campo de lo que el ser humano puede hacer de tal manera que constituye un cambio (moralmente) cualitativo con respecto al resto de intervenciones medicas.

De acuerdo con Allen Buchanan, los modos de intervención genética se pueden dividir en intervenciones directas e indirectas ${ }^{40}$. Las intervenciones directas se dividen, a su vez, en dos modos: terapia génica, "en la que se

${ }^{38}$ Sobre este tema véase: WIKLER, Daniel: "Persuasión and Coercion for Health: Ethical Issues in Government Efforts to Change Life-Styles", en SARTorius, Rolf: Paternalism, cit., pp. 35-59; y KLeInIG, John: Paternalism, Ed. Manchester University Press, Manchester, 1983, cap. V., pp. 106-142.

${ }^{39}$ Sobre los diferentes criterios usados en la asignación de recursos escasos en la práctica médica, véase: Elster, Jon y Nicolas Herpin: La ética de las decisiones médicas, Ed. Gedisa, Barcelona, 2000.

${ }^{40}$ Allen Buchanan, Dan W. Brock, Norman Daniels y Daniel Wikler: Genética y Justicia, cit., p. 5. 
insertan genes normales o deseables, bien en células somáticas (de tejido corporal) o bien en células en línea germinal (gametos -óvulos o espermatozoides- o embriones); y cirugía génica, en la que se 'desactivan' genes anormales o indeseados para que no puedan producir sus efectos específicos. Hasta el momento la terapia génica con seres humanos se ha limitado a las células somáticas. Las intervenciones indirectas igualmente se dividen en dos modos principales: farmacología genética, que consiste en "crear fármacos que sustituyan los productos químicos que serían producidos por el gen normal en un individuo que posee un gen anormal, aumentar los productos químicos de los genes normales o contrarrestar los efectos de un gen indeseable o anormal"41, y selección de embriones, que se articula en tres pasos: recogida de embriones, análisis de ADN e implante del embrión deseado. Allen Buchanan sugiere, además, una posible tercera categoría de "intervención genética", entendiendo esta expresión en sentido muy amplio, donde se incluyen dos subcategorías: las intervenciones mediante diagnóstico genético reproductivo, que consiste en usar la información genética en cuestión para tomar decisiones reproductivas (por ejemplo, la decisión de abortar un feto con síndrome de Down), y la intervención mediante prueba genética terapéutica, que se usa para prevenir o tratar enfermedades genéticas en un sujeto ya existente (por ejemplo, el tratamiento de la fenilcetonuria).

Estas intervenciones, además de los problemas comunes al conjunto de las intervenciones médicas, plantean algunos problemas específicos de justicia distributiva de notable importancia: "al acercarse más -dice Allen Buchanan- las posibilidades de realizar significativas intervenciones genéticas a gran escala en seres humanos, quizás nos veamos obligados a ampliar radicalmente nuestra concepción del ámbito de la justicia, incluyendo activos naturales además de sociales entre los bienes cuya justa distribución se supone que las instituciones deben regular, a abandonar la sencilla imagen de que la justicia trata de la distribución de bienes entre individuos que reciben su identidad independientemente del proceso de distribución, y a revisar ciertas suposiciones básicas sobre las relaciones entre la justicia, la naturaleza humana y el progreso moral" ${ }^{\prime 2}$.

\section{Paternalismo y bioética}

A partir de estas consideraciones y de las distinciones que se han hecho en el apartado anterior, creo que es posible explicar brevemente las relaciones entre el tema del paternalismo y la bioética.

\footnotetext{
${ }^{41}$ Allen Buchanan, Dan W. Brock, Norman Daniels y Daniel WikLer: Genética y Justicia, cit., p. 6.

${ }^{42}$ Allen Buchanan, Dan W. Brock, Norman Daniels y Daniel Wikler: Genética y Justicia, cit., p. 58.
} 


\section{(1) La distinción entre investigación y práctica}

El establecimiento de los límites del paternalismo médico justificado parece ser el problema fundamental de la bioética en su parte dedicada a la práctica médica clínica y asistencial, pero no tanto en lo referido a la investigación con seres humanos. Tal y como se ha trazado la distinción, la práctica se caracteriza por (1) beneficiar directamente al sujeto que es tratado y (2) por mostrar una expectativa razonable de éxito. La investigación, sin embargo, se orienta (1) a aumentar nuestro conocimiento y (2) muestra por naturaleza un alto grado de incerteza sobre los resultados. De aquí se deriva que, en el contexto de la investigación, las motivaciones de los investigadores y las justificaciones alegadas de sus acciones, cuando éstas entrañan daños o riesgos de daños para los que son objetos de investigación, no suelen ser primariamente paternalistas.

Ahora bien, esto no significa que en este contexto investigador no se plantee en absoluto el problema del paternalismo: mientras que los casos en los que se ha sometido contra su voluntad a un sujeto a experimentación no suelen ser paternalistas (porque no coincide que este mismo sujeto es el beneficiado de la intervención), los casos en los que no se permite a un sujeto someterse por propia voluntad a un experimento sí que suelen ser paternalistas, en la medida en que de lo que se trata es de proteger a este individuo de sus propias decisiones arriesgadas.

En este contexto de la investigación, conviene distinguir, no obstante, las interferencias paternalistas de aquellas que tienen por finalidad evitar algunas formas no coactivas de explotación. "Explotar a otro" es "una forma de usar a otra persona que de alguna manera es incorrecta o injusta"43. La explotación interpersonal, explica Joel Feinberg, implica siempre que a) una parte saca provecho de otra y b) por medio de "aprovecharse" de alguna característica de $B$ (la parte explotada) o de sus circunstancias. Cuando la explotación es coactiva, $A$ se aprovecha de su mayor fuerza sobre $B$. Cuando no es coactiva, el explotador juega con otras características del explotado: por ejemplo, el niño que explota el cariño de sus padres, el gorrón que explota la generosidad de un amigo, los políticos que explotan el miedo de los ciudadanos, etc. ${ }^{44}$. En el debate, por ejemplo, sobre si debe permitirse o no a los presos someterse a ensayos clínicos o sobre si estos ensayos pueden pagarse, etc., suelen entremezclarse razones paternalistas con otras que tie-

${ }^{43}$ FeinberG, Joel: "Noncoercive Exploitation”, en SARTorius, Rolf: Paternalism, cit., p. 202.

${ }^{44}$ Véase: KLeinig, John: “The Ethics of Consent”, en Kai Nielsen y Steve C. PAtTen (eds.): New Essays in Ethics and Public Policiy, Canadian Journal of Philosophy, volumen suplementario VIII, Canadian Association for Publishing in Philosophy, 1982. 
nen que ver con el problema de la explotación. Mientras que el paternalismo se centra en los riesgos asumidos por los individuos, la discusión sobre la explotación se centra en la inmoralidad que supone que unos individuos se aprovechen de otros, con independencia de si estos últimos pueden salir, en conjunto, beneficiados.

Igualmente, se plantea el problema de la explotación con respecto a prácticas experimentales o, incluso, no científicas. La prohibición en España, por ejemplo, de comercializar el medicamento Bio-Bac ${ }^{45}$ o en Estados Unidos la polémica sobre el Laetrile ${ }^{46}$ parecen justificarse más por la finalidad de impedir la explotación que en razones paternalistas (al menos, con respecto a su consumo por enfermos terminales para los que no hay tratamientos alternativos).

Tanto en lo que se refiere a la investigación como a la práctica, desde el punto de vista jurídico, una de las transformaciones más importantes promovidas por la crítica al paternalismo médico ha sido la introducción de la institución del consentimiento informado. Es en EE.UU. donde primero se utiliza esta denominación y se formalizan procedimientos para aplicar a las relaciones entre el médico y el paciente el principio de autonomía individual; en particular, se cita la sentencia de 1957 Salgo vs. Leland Stanford Jr. University Board of Trustees de la Corte de Apelación de California como la primera en la que aparece la expresión "consentimiento informado". Según esta doctrina de common law no aportar toda la información relevante sobre el tratamiento al paciente es suficiente para atribuir responsabilidad por negligencia (tort of negligence). En España, la obligación jurídica de contar con el consentimiento informado de los pacientes se introdujo en el artículo 10 de Ley 14/1986 General de Sanidad y ha sido completada y actualizada en el capítulo IV de la Ley 41/2002, de 14 de noviembre, Básica Reguladora de la Autonomía del Paciente y de Derechos y Obligaciones en Materia de Información y Documentación Clínica. Suele afirmarse la relación de jus-

${ }^{45}$ La Agencia Española del Medicamento retiró del mercado el producto conocido como Bio-Bac, que estaba siendo utilizado sin la debida licencia para el tratamiento de enfermedades tales como el cáncer, la hepatitis C o el sida. El "medicamento" fue considerado por la Agencia de "dudosa eficacia", aunque "inocuo", salvo por el abandono de tratamientos convencionales. Se calcula que el 15\% de los consumidores de Bio-Bac eran pacientes con hepatitis C, cáncer o enfermos terminales. Tras su retirada del mercado, los consumidores habituales se manifestaron públicamente exigiendo el reconocimiento de su derecho a consumirlo, llegándose a producir, incluso, el asalto de la casa de uno de los fabricantes para obtener Bio-Bac. Según algunos de ellos, la interrupción del tratamiento produjo la muerte de algunos pacientes.

${ }^{46}$ El "Laetrile" (también conocido como vitamina B-17) es un derivado de la semilla del albaricoque usado contra el cáncer por miles de estadounidenses, cuya efectividad no ha sido científicamente comprobada. La F.D.A. (Food and Drug Administration) no otorgó licencia para su uso, a pesar de lo cual, y teniendo en cuenta las protestas de consumidores y vendedores, numerosos Estados legalizaron el producto. 
tificación entre esta obligación legal y el principio constitucional del libre desarrollo de la personalidad expresado por el artículo 10 de la Constitución Española de $1978^{47}$. Obviamente, desde el punto de vista del Derecho, el consentimiento siempre ha sido relevante en cuanto a las relaciones de los individuos entre sí: el Derecho penal o el derecho privado que se ocupa de daños hacen de la inexistencia del consentimiento un elemento clave de la responsabilidad; sin embargo, la doctrina del consentimiento informado se refiere, en particular, a la prohibición de llevar a cabo intervenciones no consentidas, someter a riesgos no consentidos u ocultar información sobre riesgos a pacientes que se hallan sumergidos en una práctica institucional, la medicina, con fines terapéuticos, de investigación o mixtos ${ }^{48}$.

Por lo que respecta a la justificación del comportamiento paternalista en la práctica clínica y asistencial, a mi juicio, la vía más promisoria es, como ha señalado Ernesto Garzón ${ }^{49}$, exigir como condición necesaria de la justificación la incompetencia del sujeto tratado paternalistamente o, como ha propuesto Joel Feinberg ${ }^{50}$, que la decisión dañosa adoptada por el sujeto no sea suficientemente voluntaria. Para que esta vía de justificación sea aplicable a la resolución de problemas morales concretos, tiene que aportar, en primer lugar, una clara descripción de lo que significa "ser incompetente" o actuar de una "forma insuficientemente voluntaria" y, en segundo lugar, proporcionar criterios sobre cómo determinar que en una situación en particular una persona es incompetente o ha actuado de forma insuficientemente voluntaria. Ambas tareas son muy complejas por lo que, me parece, debemos estar preparados para hacer continuos reajustes sobre las mismas de acuerdo con nuestros principios y en relación con los casos concretos. Además, en mi opinión, algunas interferencias triviales con la autonomía orientadas a evitar daños a los pacientes podrían estar justificadas sin necesidad de mostrar la incompetencia (o involuntariedad en las elecciones) del paciente ${ }^{51}$.

\footnotetext{
${ }^{47}$ Véase: García SAn Miguel, Luis (coord.): “El libre desarrollo de la personalidad”, Ed. Universidad de Alcalá, Alcalá de Henares, 1995.

${ }^{48}$ VanDeVeer, Donald: Paternalistic Interventions, Ed. Princeton University Press, New Jersey, 1986, p. 172.

${ }^{49}$ Véase: GARZÓN VALDÉs, Ernesto: “¿Es éticamente justificable el paternalismo jurídico?”, en Doxa, cit.

${ }^{50}$ Véase: FeInBerg, Joel: Harm to Self, cit. A éste tipo de paternalismo, ejercido para evitar que las personas se dañen a sí mismas de forma insuficientemente voluntaria, le denomina Joel Feinberg "paternalismo débil", el cual contrapone al "paternalismo fuerte" ejercido sobre individuos competentes y en relación con sus decisiones suficientemente voluntarias. Para Joel Feinberg, tan sólo el paternalismo débil orientado a evitar daños físicos, psíquicos o económicos está, en algunas ocasiones, justificado. Por el contrario, el paternalismo fuerte nunca estaría justificado.

${ }^{51}$ Beauchamp y Childress ponen algunos ejemplos plausibles de estas intervenciones de "paternalismo fuerte": por ejemplo, el caso de una doctora que retiene información sobre los
} 


\section{(2) La distinción entre tratamiento y mejora}

Esta importante distinción corre paralela a la distinción entre el paternalismo que trata de evitar daños y el paternalismo, extremo o radical, que trata de promover un beneficio. En la medida en que algunas prácticas médicas paternalistas se orienten a producir una mejora y no a tratar la enfermedad, podemos encontrarnos ante supuestos de paternalismo extremo. En la práctica médica clínica y asistencial no suele darse este tipo de paternalismo, pues ésta se orienta decididamente a tratar la enfermedad. Quizás a este respecto constituya un caso particular la práctica de la psiquiatría y de la psicología. En la actualidad existe un cuerpo de conocimientos sobre diagnóstico de patologías mentales representado por el manual de diagnóstico de la Academia Americana de Psiquiatría, conocido como DSM IV ${ }^{52}$; si la actividad del psiquiatra o psicólogo se orienta a tratar un paciente "enfermo" de acuerdo con este corpus, su actividad puede describirse como tratamiento y no mejora. Sin embargo, parece que la frontera entre cliente sano y enfermo es más borrosa cuando se trata de enfermedades mentales que cuando se trata de enfermedades físicas y, de hecho, no es infrecuente que los mismos profesionales traten a clientes sanos con terapias de perfeccionamiento o mejoramiento.

Es plausible sostener que los casos de paternalismo médico justificado se darán sólo con ocasión del tratamiento de la enfermedad y orientado a evitar daños serios al paciente. El paternalismo extremo resultaría, a mi juicio, incoherente con la importancia que se le atribuye a la autonomía individual en las concepciones más plausibles de la moralidad.

\section{(3) La distinción entre prevención y curación}

Esta distinción pone de manifiesto que la relevancia del paternalismo para la bioética no se agota en el estudio del concepto y justificación del paternalismo médico. Si en el ámbito del tratamiento es indiscutible el protagonismo del médico, en el ámbito de la prevención aparecen además otros actores. En particular, es relevante el papel del Estado en la prevención de las enfermedades en general y, en particular, en la prevención paternalista de enfermedades cuya etiología son los malos hábitos de vida de los propios

\footnotetext{
resultados muy negativos de una prueba a la espera de su confirmación en una segunda prueba que se realizará en poco tiempo; o el caso de una enfermera que, contra el deseo del paciente, levanta las barras laterales de la cama para evitar que éste se caiga, puesto que la medicación que se le ha administrado puede adormecerlo. Véase: Beauchamp, Tom L. y James F. ChiLDRESS : Principios de ética biomédica, cit., pp. 267 y 268.

52 Véase: López-IBor Aliño, Juan J. (dir.): Manual Diagnóstico y Estadístico de los Trastornos Mentales, Ed. Masson S.A., Barcelona, 1999.
} 
individuos afectados. En conclusión, no sólo el paternalismo médico sino también el paternalismo estatal caería dentro del "programa" de la bioética. Lo mismo puede decirse, además, al respecto del paternalismo laboral.

Hay que advertir que entre las políticas públicas sanitarias sólo serían candidatas a la calificación de paternalistas aquellas que persiguen específicamente evitar que los individuos dañen su propia salud, mientras que ya no lo serían aquellas medidas orientadas a obtener una distribución más justa de las cargas producidas por la enfermedad o las orientadas a garantizar unas condiciones sociales en las que la salud sea posible, es decir, las orientadas a generar y proteger la salud pública.

Cuando las medidas paternalistas consisten en leyes o reglamentos que imponen prohibiciones u obligaciones por el bien de los propios destinatarios de estas prohibiciones u obligaciones (por ejemplo, la prohibición de conducir sin casco en las motos o sin cinturón de seguridad en los automóviles, la exigencia de obtener recetas médicas para la compra de medicamentos peligrosos, etc.) en la justificación de las mismas entran en juego algunas consideraciones y principios diferentes a las del caso más sencillo de un individuo ejerciendo paternalismo sobre otro. Así, por ejemplo, es plausible sostener que el principio del Derecho penal mínimo es un obstáculo muy serio para considerar justificadas a leyes penales paternalistas.

\section{(4) La distinción entre intervenciones genéticas y otras intervenciones médicas}

Quizás no sea exagerado decir que la revolución genética que estamos viviendo pudiera significar un cambio para la humanidad comparable al de la Revolución industrial y que, al igual que ocurrió con ésta, gran parte del debate ético, político y jurídico gire en el futuro en torno a cómo utilizar nuestro recién adquirido "poder genético". Al considerar la sumaria clasificación de intervenciones genéticas presentada más arriba, cabe establecer una doble distinción: en primer lugar, entre las intervenciones que agotan sus efectos en el sujeto sobre el que se realizan y aquéllas que pasan a formar parte de la herencia genética del mismo, y, en segundo lugar, entre las intervenciones que se realizan sobre potenciales seres humanos (embriones humanos) o sobre seres humanos ya existentes ${ }^{53}$. Esta frontera entre lo futuro y lo existente no coincide por supuesto con la que se suele trazar entre la competencia y la incompetencia, que serían propiedades adscribibles sólo a seres humanos existentes, aunque la condición de incompetencia comparte con la condición de "futuro ser humano" el que las decisiones que afectan

${ }^{53}$ Debo agradecer al profesor Joaquín Rueda, experto en biología celular, que me haya resuelto algunas dudas sobre la plausibilidad de esta distinción. 
al sujeto incompetente o que afectan al futuro ser deben ser tomadas por otros.

Tipos de intervenciones genéticas

\begin{tabular}{|l|c|c|}
\cline { 2 - 3 } \multicolumn{1}{c|}{} & $\begin{array}{c}\text { No pasan a formar parte } \\
\text { de la herencia genética }\end{array}$ & $\begin{array}{c}\text { Pasan a formar parte } \\
\text { de la herencia genética }\end{array}$ \\
\hline $\begin{array}{l}\text { Potenciales seres humanos } \\
\text { (embrión humano) }\end{array}$ & (1) & (2) \\
\hline Seres humanos ya existentes & (3) & (4) \\
\hline
\end{tabular}

Hasta el momento, al tratar sobre el concepto y justificación del paternalismo sólo se ha hecho referencia a intervenciones benevolentes que cabría incluir en la categoría (3) del anterior cuadro. Aquí, el que una intervención médica sea genética no parece presentar ninguna especificidad con respecto a otras intervenciones médicas: una terapia o cirugía genética en células somáticas o el tratamiento con farmacología genética pueden constituir supuestos de paternalismo médico dependiendo del modo de la intervención. Tan sólo me parece importante destacar que estas intervenciones genéticas son, en muchas ocasiones, terapias experimentales, lo que hace más difícil justificar una aplicación paternalista de las mismas, puesto que las probabilidades de éxito son inciertas. El riesgo de que la aplicación del tratamiento tenga peores consecuencias que la no aplicación hace que la acción paternalista se oriente más bien a no permitir al enfermo someterse a la terapia o, al menos, a elevar notablemente el estándar del consentimiento válido para permitírselo.

Los casos que caerían en la categoría (1) ya suponen un importante cambio con respecto a los tipos de paternalismo que hasta ahora hemos considerado, ya que se ha dado por supuesto que el sujeto $B$ de la relación paternalista es un sujeto existente ${ }^{54}$. Además, el concepto de paternalismo, como hemos visto, parece exigir no sólo un sujeto que meramente existe, sino que además es portador de algunas preferencias, aunque esto no implica, en mi opinión, que sea necesariamente competente. A juicio de Joel Feinberg, la protección y cuidado de quienes no pueden hacerlo por sí mismos es "paternalismo" en un sentido diferente al "paternalismo" ejercido sobre personas que "todavía son bastantes capaces de decidir por sí mismas" 55 . Si se acepta la propuesta de Joel Feinberg, las acciones benevolentes con respecto a fu-

${ }^{54}$ Para que una intervención genética sobre el embrión limite sus efectos al futuro ser y no pase a su herencia genética, debería hacerse una vez rebasada la fase de blastocisto y no afectar a la células gonadales.

${ }^{55}$ FeinBerg, Joel: Harm to Self, cit., p. 6. 
turos sujetos sería un caso límite de este "paternalismo presumiblemente no censurable" $y$, en definitiva, se trataría de otro paternalismo diferente al que nos ocupa en este artículo. Sin embargo, cabría optar por tratar de dar un concepto unitario de paternalismo que englobe desde el caso en que el paternalista impone un beneficio a un adulto competente contra su voluntad hasta el caso en que se toma una decisión benevolente sobre el futuro de un ser todavía no existente, de manera que algunas de las intervenciones incluidas en esta categoría (1) podrían ser correctamente descritas como paternalistas. Esta segunda opción nos desafía a dar con una caracterización más amplia del elemento negativo de la acción paternalista, el modo de la acción. Quizás, una vía para esta ampliación sería considerar que la acción paternalista se produce siempre que alguien toma una decisión con intención de beneficiar a otro (sea un ser existente o un futuro ser) que limitará oportunidades significativas de la vida de éste, con independencia de si éste estaba o no en condiciones de elegir por sí mismo (lo cual es relevante para la justificación de la acción paternalista). Desde este punto de vista, podríamos considerar como casos de paternalismo, y aplicar a los mismos nuestro conocimiento sobre cómo éstos pueden ser o no justificados, supuestos tan llamativos como el de abortar a un feto que padecerá, según un análisis genético, una enfermedad incurable, no tratable e "incompatible con una vida digna" 56 .

Las otras dos categorías restantes, (2) y $(4)^{57}$, podrían plantear problemas de paternalismo análogos a los de (1) y (2), pero, además, suscitan un problema diferente que es el de la eugenesia. Por ello, me parece importante distinguir entre las motivaciones paternalistas y las propiamente eugenésicas. "Eugenesia" es un término que guarda ciertos parecidos con el de "paternalismo". Al igual que este último, su origen se remonta al último tercio del siglo XIX, cuando Francis Galton lo definió como "la ciencia de mejorar el linaje, no sólo mediante un apareamiento juicioso, sino mediante todo aquello que tienda a dar a las razas o líneas sanguíneas más adecuadas mayores oportunidades de prevalecer sobre las menos aptas de las que de otra manera habrían tenido" 58 . El daño que fundamentalmente busca evitar la eu-

${ }^{56}$ Sobre la problemática moral de estos casos, véase: Allen Buchanan, Dan W. Brock, Norman Daniels y Daniel WikLeR: Genética y Justicia, cit., cap. VI.

${ }^{57}$ Dentro de la categoría (2) caería la intervención genética sobre un embrión en fase de blastocisto: cualquier modificación genética de las células madre "totipotentes" conllevaría una modificación del genoma de todas las futuras células del individuo, incluídas las gonadales. Igualmente, entraría en esta categoría la modificación del genoma de las celulas gonadales del embrión, una vez superada la fase de blastocisto. En la categoría (4) caería la intervención genética sobre un adulto que modificara el génoma de todas sus células, incluidas las gonadales, y, también, el caso en que sólo se modificaran su células gonadales.

${ }^{58}$ Allen Buchanan, Dan W. Brock, Norman Daniels y Daniel Wikler: Genética y Justicia, cit. p. 28. 
genesia es el de la degeneración, ya sea de la especie humana en general, de la raza o de la etnia. Entre las medidas eugenésicas se suele distinguir entre medidas positivas (por ejemplo, los premios a las familias que satisfacen el modelo de la raza) o negativas (por ejemplo, la esterilización involuntaria de sujetos no deseables). Es fácil advertir que algunas medidas eugenésicas negativas pueden coincidir en el modo empleado con supuestos de paternalismo, por lo que es importante no perder de vista la diferente finalidad que corresponde a estas intervenciones. Considerar el caso de la esterilización de deficientes mentales puede ayudar a ver con claridad la diferencia (aunque, por supuesto, la esterilización no es una intervención genética).

Desde el punto de vista del paternalismo, es plausible sostener que en lugar de impedir desde el Estado, como se hace con frecuencia, que un deficiente mental tenga relaciones sexuales por medio de prohibir a todos los demás tenerlas con él, sería más beneficioso para el deficiente posibilitarle estas relaciones pero tratando de atenuar los riesgos vinculados a las prácticas sexuales: por ejemplo, dadas sus circunstancias, el riesgo de la procreación $^{59}$. Por ello, cabría ensayar una justificación paternalista de la esterilización de deficientes mentales. Diferente sería la justificación eugenésica, según la cual, es legítimo para las autoridades impedir o dificultar que información genética defectuosa se extienda y en la que el individuo es sacrificado por el grupo. Para el paternalista el objetivo es evitar daños al propio sujeto esterilizado, por lo que debe evaluar todas las consecuencias que esta medida tenga para el deficiente. De ahí que si la esterilización no es una solución frente a las enfermedades de transmisión sexual o frente al riesgo de explotación sexual por otros, en última instancia puede encontrar razones para no esterilizar y buscar otras alternativas.

Una última reflexión para finalizar. Hay que reconocer que el paternalismo es un problema difícil, tanto por lo que se refiere al concepto como a la justificación. A veces, el paternalismo parece abarcarlo todo (hasta el Estado mismo sería una autoridad paternalista), otras veces, parece no referirse a nada (fuera de la familia no hay verdadero paternalismo y en la familia no se trata de paternalismo sino de cuidado paternal). Con frecuencia el paternalismo se presenta como algo necesariamente injustificado, a veces conscientemente y otras veces de forma inadvertida. Difícilmente se sustraen los

${ }^{59}$ La noción de riesgo se refiere a la probabilidad y gravedad de un daño. Un embarazo en el caso de mujeres con deficiencias mentales, dadas sus circunstancias, puede conllevar serios daños para ellas mismas. En el caso de los hombres los daños serán de una naturaleza diferente y plausiblemente de menor gravedad: no relacionados con la gestación pero sí con la imposibilidad de afrontar la paternidad. 
autores y lectores a la fuerte carga peyorativa del término "paternalismo". Sin embargo, a mi juicio, no sólo hay inconvenientes en el uso de este lábil término de "paternalismo". Los comportamientos paternalistas son siempre moralmente sospechosos y el término generalmente peyorativo es una señal de alarma moral. Vale la pena mantener esta señal de alarma, y esto es un reconocimiento a la importancia que merece el respeto por la autonomía individual, sin por ello renunciar a enfrentar los conflictos reales que se producen entre la autonomía y el bienestar individuales. Confío en que este artículo contribuya a evitar, particularmente en el ámbito de la bioética, dos importantes errores: creer, en primer lugar, que el paternalismo nunca está justificado y, en segundo lugar, creer que el comportamiento paternalista no necesita justificarse.

\section{Bibliografía}

AtienZa, Manuel: “Juridificar la bioética”, en Vázquez, Rodolfo (comp.): Bioética y Derecho (Fundamentos y problemas actuales), Ed. Itam-FCE, México.

Babiano Mora, J.: Paternalismo industrial y disciplina fabril en España, 19381958, Ed. CES, Madrid, 1998.

Beauchamp, Tom L. y James F. Childress: Principios de Ética Biomédica, Ed. Masson, Barcelona, 2002.

Betegón, Jerónimo y Juan Ramón de PÁramo (eds.): Derecho y Moral. Ensayos analíticos, Ed. Ariel, Barcelona, 1990.

BobBio, Norberto: Locke e il diritto naturale, ed. G. Giappichelli, Torino, 1963.

Buchanan, Allen, Dan W. Brock, Norman Daniels y Daniel Wikler: Genética y Justicia, Ed.Cambridge University Press, Madrid, 2002.

Boorse, Christopher : "On the Distinction between Disease and Illness", Philosophy and Public Affairs, 5 (1975), núm. 1.

- "Wright on Functions", en Philosophical Review, 85(1976), núm. 1.

- "Health as a Theoretical Concept", en Philosophy of Science, 44 (1977).

Culver, Charles M. y Bernard Gert: Philosophy in Medicine (Conceptual and Ethical Issues in Medicine and Psychiatry), ed. Oxford University Press, N.Y., 1982.

Daniels, Norman: Just Health Care, Cambridge University Press, Cambridge, 1985.

Dworkin, Gerald: "Paternalism", en Wasserstrom, R. A. (ed.): Morality and the Law, Ed. Wadsworth Publishing Co., Belmont, 1971.

ELSTER, Jon: Ulises y las Sirenas (Estudios sobre racionalidad e irracionalidad), Ed. FCE, México, 1997.

Elster, Jon y Nicolas Herpin: La ética de las decisiones médicas, Ed. Gedisa, Barcelona, 2000.

ELSTER, Jon: Ulises desatado (Estudios sobre racionalidad, precompromiso y restricciones), Ed. Gedisa, Barcelona, 2002.

Feinberg, Joel: "Legal Paternalism", en Canadian Journal of Philosophy 1, nº 1 (1971). 
Feinberg, Joel: The Moral Limits of the Criminal Law. Harm to Self, Ed. Oxford University Press, N.Y. 1986.

- Harmless Wrong-Doing, Ed. Oxford University Press, N.Y., 1990.

FeinBerg, Joel y Jules Coleman (eds.): Philosophy of Law, Ed. Wadsworth, Belmont, 2000.

García San Miguel, Luis (coord.): "El libre desarrollo de la personalidad", Ed. Universidad de Alcalá, Alcalá de Henares, 1995.

GARZÓN VALDÉs, Ernesto: “¿Es éticamente justificable el paternalismo jurídico?”, en Doxa, 5 (1988).

- "Intervencionismo y paternalismo", en Garzón Valdés, Ernesto: Derecho, ética y política, Ed. CEC, Madrid, 1993.

- “Puede la razonabilidad ser un criterio de corrección moral?”, en Doxa, 21 (1998), vol. II.

Gert, Bernard: The Moral Rules (A New Rational Foundation for Morality), Ed. Harper \& Row, Nueva York, 1970.

Gert, Bernard y Charles M. Culver: "Paternalistic Behavior", Philosophy \& Public Affairs 6 (1976), vol. 1.

- "The Justificaction of Paternalism", Ethics 89 (1979).

Gert, Bernard, Charles M. Culver y K. Danner Clouser: Bioethics. A Return to Fundamentals, Ed. Oxford University Press, N.Y., 1997.

Gracia, Diego: "Historia de la ética médica", en Vilardell, Francisco: Ética y medicina, Ed. Espasa, Madrid, 1988.

- Procedimientos de decisión en ética clínica, Ed. Eudema, Madrid, 1991.

Hart, H.L.A.: Law, Liberty and Morality, Ed. Stanford University Press, 1963.

- "Rawls on Liberty and Its Priority", en Daniels, Norman (ed.): Reading Rawls, Ed. Basic Books, Nueva York, 1975.

HochschiLD, Adam: El fantasma del rey Leopoldo (Codicia, terror y heroísmo en el África colonial), Ed. Península, Barcelona, 2002.

Jonsen, Albert R. y Stephen Toulmin: The Abuse of Casuistry: A History of Moral Reasoning, Ed. University of California Press, Berkeley, 1988.

Kleinig, John: “The Ethics of Consent”, en Kai Nielsen y Steve C. Patten (eds.): New Essays in Ethics and Public Policiy, Canadian Journal of Philosophy, volumen suplementario VIII, Canadian Association for Publishing in Philosophy, 1982.

- Paternalism, Ed. Manchester University Press, Manchester, 1983.

Locke, John: Segundo ensayo sobre el gobierno civil, Ed. Austral, Madrid, 1997.

LópeZ-IBor Aliño, Juan J. (dir.): Manual Diagnóstico y Estadístico de los Trastornos Mentales, Ed. Masson S.A., Barcelona, 1999.

Nino, C.S.: Ética y derechos humanos, Ed. Ariel, Barcelona, 1989.

Rawls, John: Teoría de la Justicia, Ed. Fondo de Cultura Económica, México, 1997.

Rescher, Nicholas: Welfare: The Social Issue in Philosophical Perspective, Ed. University of Pittsburgh Press, Pittsburgh, 1972.

SArtorius, Rolf: Paternalism, Ed. University of Minnesota Press, Minneapolis, 1987. 
Sierra Álvarez, José, El obrero soñado. Ensayo sobre el paternalismo industrial (Asturias, 1860-1917), Ed. Siglo XXI, Madrid, 1990.

Toulmin, Stephen: "De qué manera la medicina le salvó la vida a la ética", en Análisis filosófico, vol. XVII, 2(1997).

VanDeVeer, Donald: Paternalistic Interventions, Ed. Princeton University Press, New Jersey, 1986.

WIKLER, Daniel: "Persuasión and Coercion for Health: Ethical Issues in Government Efforts to Change Life-Styles", en Sartorius, Rolf: Paternalism, Ed. University of Minnesota Press, Minneapolis, 1987. 